PROCEEDINGS OF THE

AMERICAN MATHEMATICAL SOCIETY

Volume 130, Number 9, Pages 2797-2801

S 0002-9939(02)06391-8

Article electronically published on February 4, 2002

\title{
ANTI-SYMPLECTIC INVOLUTIONS WITH LAGRANGIAN FIXED LOCI AND THEIR QUOTIENTS
}

\author{
YONG SEUNG CHO AND DOSANG JOE
}

(Communicated by Ronald A. Fintushel)

\begin{abstract}
We study the lagrangian embedding as a fixed point set of antisymplectic involution $\tau$ on a symplectic 4-manifold $X$. Suppose the fixed loci of $\tau$ are the disjoint union of smooth Riemann surfaces $X^{\tau}=\dot{U} \Sigma_{i}$; then each component becomes a lagrangian submanifold. Furthermore, if one of the components is a Riemann surface of genus $g \geq 2$, then its quotient has vanishing Seiberg-Witten invariants. We will discuss some examples which allow an anti-symplectic involution with lagrangian fixed loci.
\end{abstract}

\section{INTRODUCTION}

To find a lagrangian embedding in a compact symplectic manifold is a quite subtle problem. In particular, in a 4-dimensional symplectic manifold, the existence of a compact Riemann surface of higher genus as a lagrangian submanifold is highly nontrivial. In fact, it should have a positive second betti number, $b_{2}^{+}$, which is greater than two. In this paper, we will discuss a lagrangian embedding as a fixed loci of anti-symplectic involution. Even though every lagrangian submanifold cannot be realized in this way, there is no known way to construct a lagrangian embedding so far. It is easily proved that every embedded lagrangian surface has minimal genus among its homology class via the Seiberg-Witten theory. Since every lagrangian surface can be realized as a symplectic submanifold for a new symplectic form by small deformation $\mathrm{G}$, from the fact that every symplectic surface has such a genus minimizing property [M] MST, OS], it has a minimal genus. In general, this can be proved by a simple argument via the so-called adjunction inequality which says that $2 g(\Sigma)-2 \geq \Sigma^{2}$ where $g$ is the genus of the embedded surface $\Sigma$ and $\Sigma^{2}$ indicates the self-intersection number. It is checked that any lagrangian embedding equalizes the inequality since the normal bundle of lagrangian embedding $\left(i: \Sigma_{g} \hookrightarrow\right.$ $X)$ is naturally isomorphic to the cotangent bundle of the surface; it implies that the self-intersection number of $\Sigma$ is minus the Euler characteristic of $\Sigma$, i.e. $\Sigma^{2}=$ $\Sigma_{g} \cdot \Sigma_{g}=2 g-2$.

Moreover suppose there exists an embedded Riemann surface $\Sigma_{g}$ of $g \geq 2$ in $X$ whose self-intersection number is $2 g-2$. Then one can show that $X$ must be of Seiberg-Witten simple type, i.e. every basic class induces an almost-complex

Received by the editors October 13, 1999 and, in revised form, April 18, 2001.

2000 Mathematics Subject Classification. Primary 57N13, 57N35, 57R57.

The first author was supported in part by KOSEF grant \#1999-2-101-002-5.

The second author was supported in part by KOSEF grant \#2000-2-10100-002-3.

This work was supported in part by BK21 project. 
structure OS, FS1]. On the other hand, if $X$ contains a Riemann surface of genus $g$ whose intersection number is strictly greater than $2 g-2$, then $X$ has all vanishing Seiberg-Witten invariants. This observation can be easily proved by the blow-up formula due to R. Fintushel and R. Stern [FS1, FS2]. Therefore we can show that if the fixed loci of anti-symplectic involution $\tau$ on $X$ contain a lagrangian surface of higher genus $\geq 2$ as a component, then its quotient has vanishing SW invariant. However, it does not necessarily imply that the quotient does not have symplectic structure unless $g \geq 2$. There is an algebraic quartic (degree 4) hypersurface in $\mathbb{C} P^{3}$, i.e. K3 surface, which has a lagrangian torus as fixed loci of the canonical anti-holomorphic (anti-symplectic) involution whose quotient has a natural symplectic structure. In the next section, we review the topological restriction of the existence of lagrangian embedding and anti-symplectic involution, we construct the examples of lagrangian submanifolds in the smooth hypersurfaces in $\mathbb{C} P^{3}$ as fixed loci of the canonical anti-symplectic involution, and we discuss a way of constructing lagrangian embedding for the special case. For the purpose of this exposition, we shall omit the definition and details related to the Seiberg-Witten invariant. One can refer to [M, T, W] [FS1, [FS2] for all the facts and theorems from the Seiberg-Witten theory and its application to the smooth, in particular symplectic, 4-manifolds.

\section{Anti-SympleCtic INVOLUtion With LAGRANGIAN FIXED LOCI}

Let $(X, \omega)$ be a closed, symplectic 4-manifold. A diffeomorphism $\tau$ on $(X, \omega)$ is anti-symplectic if and only if $\tau^{*} \omega=-\omega$. The involution means that $\tau$ has diffeomorphism of order 2, i.e. $\tau^{2}=$ identity on $X$. Notice that $\tau \in \operatorname{Diff}^{+}(X)$ is an orientation preserving diffeomorphism, where the orientation of $X$ is the canonical one induced by the symplectic form. In [C], the first author shows that for a symplectic 4-manifold $X$ with $c_{1}(X)^{2}>0$ and $b_{2}^{+}(X)>3$ and for a $\tau$ that is a free anti-symplectic involution on $X$, then the Seiberg-Witten invariants on the quotient $X / \tau$ vanishes identically. Let $i: \Sigma \hookrightarrow(X, \omega)$ be a lagrangian embedding if $i$ is an embedding and $\left.i^{*}(\omega)\right|_{\Sigma} \equiv 0$. By the lagrangian neighborhood theorem [MS] due to Weinstein, where the normal bundle $N_{\Sigma / X}$ is isomorphic to the cotangent bundle of $\Sigma$, we have that the self-intersection number is equal to $2 g-2$. Suppose a symplectic 4-manifold $X$ has lagrangian surface of genus greater than or equal to two; then since both $\omega$ and $\Sigma$ have positive square and $\omega \cdot \Sigma=0$ (here we abuse the notation for $P D(\Sigma) \in H^{2}(X)$ as $\left.\Sigma\right), X$ has the positive second betti number greater than 2 , i.e. $b_{2}^{+}(X) \geq 2$. Otherwise one can draw a contradiction by considering the positive cone structure of the intersection pairing of $\left\langle H^{2}(X ; \mathbb{R}), \cdot\right\rangle$. Hence one can show that Enriques surfaces or rational ruled surfaces, for example, do not allow an embedded lagrangian surface of genus $\geq 2$.

We are going to study such a lagrangian embedding as fixed loci of an antisymplectic involution. It is easy to show that the two-dimensional fixed point sets, $X^{\tau}$, of anti-symplectic involution $\tau,\left.\omega\right|_{X^{\tau}}=\left.\tau^{*} \omega\right|_{X^{\tau}}=-\left.\omega\right|_{X^{\tau}}=0$, become lagrangian submanifolds. In the next section, we will provide the examples of such involution on the complex hypersurface in $\mathbb{C} P^{3}$ as a natural complex conjugation. Before getting into the discussion of the examples, we want to make a statement on what can be proved about the anti-symplectic quotient. The following theorem was mentioned by Mikhalkin on Kirby's problem 4.104 and we cannot find the proof of it, so we will fill out the line of the argument. 
Proposition 2.1. Let $(X, \omega)$ be a closed, smooth symplectic 4-manifold and let $\tau$ be an anti-symplectic involution on $(X, \omega)$ with fixed loci $X^{\tau}=\dot{U} \Sigma$ as a disjoint union of lagrangian surfaces. If one of the components of $X^{\tau}$ is a Riemann surface of genus $g \geq 2$ and $b_{2}^{+}(X / \tau) \geq 2$, then $X / \tau$ has vanishing Seiberg-Witten invariants.

Proof. Let $\pi: X \rightarrow X / \tau$ be the projection map and let $\Sigma_{g}$ be the component of $X^{\tau}$ of genus $g$. Let $\bar{\Sigma}_{g}$ be the image of $\Sigma, \pi(\Sigma)$. Since $\Sigma_{g} \cdot \Sigma_{g}=2 g-2$, $\bar{\Sigma}_{g}$ has a self-intersection number $4 g-4$ which is strictly greater than $2 g-2$ for $g \geq 2$. Since it violates the adjunction inequality, $X / \tau$ has vanishing Seiberg-Witten invariants.

\section{EXAmples}

In this section, we will investigate a way of constructing anti-symplectic involutions on some symplectic 4-manifolds.

1) Hypersurfaces in $\mathbb{C} P^{3}$. We will show a way to find the equations of a smooth algebraic hypersurface of degree $d$ in $\mathbb{C} P^{3}$ which contains a lagrangian embedding of higher genus. Since every smooth hypersurface $X_{d}$ of degree $d$ is symplectically isomorphic to each other, by Moser's deformation theorem, every smooth hypersurface allows such a lagrangian embedding. To show a lagrangian embedding of higher genus $g \geq 2$ on hypersurface $X_{d}$ of degree $d$ in $\mathbb{C} P^{3}, d$ must be greater than or equal to 4 . Since if $d<4$, then $b_{2}^{+}\left(X_{d}\right)=1$.

Consider $X_{d}(F)=\left\{(x: y: z: w) \in \mathbb{C} P^{3} \mid F(x, y, z, w)=0\right\}$ where $F$ is a homogeneous polynomial of degree $d$. Suppose the defining equation $F$ has real coefficients, i.e. $F \in \mathbb{R}[x, y, z, w]$; then there is a natural anti-holomorphic involution. This also becomes an anti-symplectic involution for the Kähler form which is induced by the restriction of the Fubini-Study metric on $\mathbb{C} P^{3}$ such as

$$
\begin{gathered}
\tau: X_{d}(F) \longrightarrow X_{d}(F), \\
(x: y: z: w) \longrightarrow(\bar{x}: \bar{y}: \bar{z}: \bar{w}) .
\end{gathered}
$$

The fixed locus of this involution, $X_{d}(F)^{\tau}$, is the set of the real solutions of the equation

$$
X^{\tau}=X \cap \mathbb{R} P^{3},
$$

where $\quad \mathbb{R} P^{3}=\left\{(x: y: z: w) \mid \quad \exists \rho \in \mathbb{C}, \quad \rho \cdot(x, y, z, w) \in \mathbb{R}^{4}\right\}$. We will consider the cases separately depending on the parity of degree $d \geq 4$.

- $d=$ even case.

Let us choose a sequence of $n$ real numbers such that $a_{1}<a_{2}<\cdots<a_{g}$ and $\left|a_{i}-a_{i+1}\right|>2 r$. Let $f_{0}=R^{2}-x^{2}-y^{2}$ and $f_{i}=\left(x-a_{i}\right)^{2}+y^{2}-r^{2}$ where $R>\max \left(\left|a_{1}\right|,\left|a_{g}\right|\right)+r$. Then the equation for the Riemann surface of genus $g$ is

$$
\Sigma_{g}=\left\{(x, y, z) \mid z^{2 g+2}=f_{0} \cdot f_{1} \cdots f_{g}\right\} \subset \mathbb{R}^{3} .
$$

Since the region $O=\left\{(x, y) \mid f_{0} \cdot f_{1} \cdots f_{g} \geq 0\right\}$ is a disc with $g$ holes in the plane $\mathbb{R}^{2}$, it follows that the projection $p: \Sigma \longrightarrow O$ is a branched 2:1 covering with $g+1$ circles as branch the locus. Projectivize this equation in $\mathbb{C} P^{3}$,

$$
F=Z^{2 g+2}-F_{0} \cdot F_{1} \cdots F_{g}
$$

where $F_{0}=R^{2} W^{2}-X^{2}-Y^{2}, F_{i}=\left(X-a_{i} W\right)^{2}+Y^{2}-r^{2} W^{2}$ for $1 \leq i \leq g$.

Let $X_{d}(F) \subset \mathbb{C} P^{3}$ be the complex hypersurface of degree $d=2 g+2$. Since $X(F) \cap\{W=0\}=\left\{(x, y, z) \mid z^{2 g+2}+\left(x^{2}+y^{2}\right)^{g+1}=0\right\}, X(F)$ has no real solutions 
on the hyperplane $\{W=0\}$, it follows that $X(F) \cap \mathbb{R} P^{3}=\Sigma_{g}$. Unfortunately, since this complex hypersurface is not smooth, we have to deform the given equation a little bit. Let $F_{\epsilon}=F+\epsilon X^{d}$. It is easily checked that $X\left(F_{\epsilon}\right)$ is a smooth complex hypersurface for small positive $\epsilon$. The real solution for the $X\left(F_{\epsilon}\right)$ is $\Sigma_{\epsilon}=$ $\left\{(x, y, z) \mid z^{2 g+2}=f_{0} \cdot f_{1} \cdots f_{g}-\epsilon x^{2 g+2}\right\}$. Since the region $O_{\epsilon}=\left\{(x, y) \mid f_{0} \cdot f_{1} \cdots f_{g} \geq\right.$ $\left.\epsilon x^{2 g+2}\right\}$ is diffeomorphic to $O$ for small $\epsilon$, the $\Sigma_{\epsilon}$ is diffeomorphic to $\Sigma_{g}$.

- $d=$ odd case.

Unlike the even degree case, the real smooth algebraic hypersurface of odd degree in $\mathbb{R} P^{3}$, which is a fixed locus of canonical complex conjugation of its complexification, does not lie in a compact subset of the real affine piece $\left(\cong \mathbb{R}^{3}\right)$. Actually, it intersects nontrivially with any hyperplane in $\mathbb{R} P^{3}$.

Let $F_{0}, \cdots, F_{g}$ be the quadratic equations defined as above and let $a<-R$ be a real number. Let $F=Z^{2 g+2} \cdot W-(X-a W) \cdot F_{0} \cdots F_{g}$. Then we can show that the set of real solutions of $F$, i.e., $X^{\tau}(F)=X_{d}(F) \cap \mathbb{R} P^{3}$, is $\mathbb{R} P^{2} \cup \Sigma_{g}$. On the affine piece,

$$
\begin{aligned}
X^{\tau}(F) \cap\{W \neq 0\} & =\left\{(x, y, z) \mid z^{2 g+2}=(x-a) \cdot f_{0} \cdots f_{g}\right\} \\
& \cong \mathbb{R}^{2} \cup \Sigma_{g},
\end{aligned}
$$

which is a $2: 1$ covering of a $g$ punctured disc and half plane with $g+1$ circles and one line as the branch locus. The points at infinity of $X^{\tau}(F)$ are a real projective line, i.e.

$$
\begin{aligned}
X^{\tau}(F) \cap\{W=0\} & =\left\{(X: Y: Z: 0) \mid X \cdot\left(X^{2}+Y^{2}\right)^{g+1}=0\right\} \\
& =\{X=0, W=0\} \cong S^{1} .
\end{aligned}
$$

At the points at infinity of $X^{\tau}(F)$, a circle is attached to the $\mathbb{R}^{2}$, hence $X^{\tau}(F) \cong$ $\mathbb{R} P^{2} \dot{\cup} \Sigma_{g}$. As we have seen in the first case, the complex hypersurface $X(F)$ is not smooth, so we can deform this equation to $F_{\epsilon}=F+\epsilon X^{2 g+3}$ for small $\epsilon$. Then one can easily show that $X^{\tau}\left(F_{\epsilon}\right) \cong X^{\tau}(F)$ and $X\left(F_{\epsilon}\right)$ is smooth.

It can be checked that the quotient by this involution has positive second betti number greater than $2, b_{2}^{+}\left(X_{d} / \tau\right)=\frac{1}{2}\left(b_{2}^{+}\left(X_{d}\right)-1\right) \geq 2$, if the degree $d$ is greater than 5. So far, we have constructed the lagrangian surface of genus $g=\left[\frac{d-2}{2}\right]$ for the hypersurface of degree $d$ in $\mathbb{C} P^{3}$. This gives rise to the question of whether every algebraic surface with nonzero geometric genus contains a lagrangian surface of genus $\geq 2$.

Remark 3.1. Following this line of reasoning, we can find a complex hypersurface of degree $d$ whose real part is a disjoint union of lagrangian surfaces $\left(\dot{\cup} \Sigma_{g_{i}}\right.$ or $\mathbb{R} P^{2} \cup \Sigma_{g_{i}}$ respectively depending on the parity of $d$ ) by taking various configurations of quadratic equations.

Maybe the next case is the most typical way of constructing an anti-symplectic involution on the product of two Riemann surfaces with same genus.

2) $X=\left(\Sigma_{g} \times \Sigma_{g}, \omega \oplus \omega\right)$. Let $f: \Sigma_{g} \rightarrow \Sigma_{g}$ be an anti-symplectic diffeomorphism of $\Sigma_{g}$, i.e. $f^{*} \omega=-\omega$. Then we can define an anti-symplectic involution $\tau_{f}$ associated with $f$ such that

$$
\begin{gathered}
\tau_{f}: \Sigma_{g} \times \Sigma_{g} \longrightarrow \Sigma_{g} \times \Sigma_{g}, \\
\quad(x, y) \longrightarrow\left(f^{-1}(y), f(x)\right)
\end{gathered}
$$


Clearly, $\tau_{f}^{2}=i d$ and $\tau_{f}^{*}(\omega \oplus \omega)=-(\omega \oplus \omega)$. We can easily show that a fixed point set of $\tau_{f}$ is the graph of the $f$, i.e.

$$
\left(\Sigma_{g} \times \Sigma_{g}\right)^{\tau_{f}}=\{(x, y) \mid y=f(x)\} \cong \Sigma_{g} .
$$

By the Hirzebruch signature theorem, we can get $b_{2}^{+}\left(X / \tau_{f}\right)=\frac{1}{2}\left(b_{2}^{+}(X)-1\right)=g^{2} \geq$ 2 if the genus $g \geq 2$.

\section{ACKNOWLEDGEMENT}

We would like to thank R. Fintushel for pointing out the known facts from the Seiberg-Witten theory which were stated as propositions in the first draft and to the referee for suggesting to us a way of better writing.

\section{REFERENCES}

[C] Y.S. Cho, Finite group actions on 4-manifolds, Jour. of the Australian Math. Soc. Series $A, 65: 1-10,1998$. MR 2000d:57052

[FS1] R. Fintushel and R. Stern, 4-manifolds and the immersed Thom-conjecture, Turkish J. Math 19, 145-157, 1995. MR 96j:57036

[FS2] R. Fintushel and R. Stern, Rational blow downs of smooth 4-manifolds, J. Diff. Geom 46, 181-235, 1997. MR 98j:57047

[G] R. Gompf, A new construction of symplectic manifolds, Ann. of Math 142, 527-595, 1995. MR 96j:57025

[M] John W. Morgan, The Seiberg-Witten equations and applications to the topology of smooth four-manifolds, volume 44 of Mathematical Notes, Princeton University Press, Princeton, NJ, 1996. MR 97d:57042

[MS] Dusa McDuff and Dietmar Salamon, Introduction to Symplectic Topology, Clarendon Press, Oxford, 1995. MR 97b:58062

[MST] John W. Morgan, Zoltán Szabó, and Clifford Henry Taubes, A product formula for the Seiberg-Witten invariants and the generalized Thom conjecture, J. Differential Geom., 44(4):706-788, 1996. MR 97 m:57052

[OS] P.B. Osváth and Z. Szabó, The symplectic Thom conjecture, Ann. of Math, to appear.

[T] Clifford Henry Taubes, The Seiberg-Witten invariants and symplectic forms, Math. Res. Lett., 1(6):809-822, 1994. MR 95j:57039

[Wa] S. Wang, A vanishing theorem for Seiberg-Witten invariants, Math. Res. Lett., 2(1):305310, 1995. MR 96c:57056

[W] Edward Witten, Monopoles and four-manifolds, Math. Res. Lett., 1(6):769-796, 1994. MR 96d:57035

Department of Mathematics, Ewha Women's University, Seoul 120-750, Korea

E-mail address: yescho@mm.ewha.ac.kr

Department of Mathematics, Pohang University of Science and Technology, Pohang 790-784, KOREA

E-mail address: joe@euclid.postech.ac.kr 